\title{
BUILDING UP A BEARING COLUMN OF AGEING VISCOELASTIC MATERIAL UNDER SIMULTANEOUS ACTION OF LOADING
}

\author{
D. A. Parshin *
}

\begin{abstract}
A mechanical problem on tracing the stress-strain state evolution of a sufficiently high conical column bearing a variable central axial loading on its upper end and being simultaneously built up by means of additive layer-by-layer piecewise continuous augmenting with new viscoelastic ageing material over the side surface is investigated. The acting axial force magnitude, the radius of the increasing bearing end, and the slope angle of the side surface are arbitrarily changing during the considered augmenting process. The closed-form analytical solution of the corresponding non-classical boundary-value problem is obtained.
\end{abstract}

\section{Keywords: Ageing, Viscoelasticity, Bearing Column, Building Up, Stress-State State.}

\section{Problematics of the study}

Additive manufacturing processes are accompanied by increase of solids in size due to adding new material layers to their surfaces. A characteristic feature of such processes is the fact that the solid does not exist in its final configuration before the start of its deforming, as it is always supposed in the classical solid mechanics. The additively manufactured solids keep being formed in course of the deformation process. So the mechanical analysis of additive processes is to take into account simultaneous acting external loads being actual in the modelled technological process, including those acting on the attached additional material, and the mechanical peculiarities of gradual attaching this material to the solid surface. Such account cannot be correctly carried out within the framework of classical solid mechanics, even if the traditional equations and boundary conditions are formulated for a time variable spatial domain. This is explained by absence of any unstressed configuration for the entire additive-manufactured solid. There is accordingly no any reference configuration of the whole solid in relation to which strain measures can be traditionally defined.

Thus, the problems on mechanical modelling for additive-manufactured solids constitute a special class of problems of solid mechanics and possess a number of non-classical effects. One can familiarize the approaches of the corresponding field of solid mechanics and also results achieved by Russian science school in mechanics of additive processes founded by Professor A. V. Manzhirov, e.g., on the basis of the papers Manzhirov and Parshin (2015a, 2015b, 2016), Bychkov et al. (2017), Parshin (2017a, 2017b, 2018), Parshin and Manzhirov (2018).

The present paper is devoted to a non-classical quasi-static problem for an additively manufactured solid with integral satisfying static boundary conditions on a part of the solid surface being expanding due to the additional material influx in the course of the solid formation. The material used in the solid formation process exhibits rheological properties, namely the properties of deformation heredity and ageing. Additive processes of forming solids of such materials are quite difficult to model, because the in time evolving deformation reaction of the being formed solid to the applied load continuously interacts with the mechanical response to the attaching new material elements to the solid, and also the formed solid

Senior Researcher, Assoc. Prof. Dmitry Alexandrovich Parshin, PhD.: Ishlinsky Institute for Problems in Mechanics of the Russian Academy of Sciences, Laboratory of Modelling in Solid Mechanics, Vernadskogo Ave. 101 Bldg 1, 119526, Moscow; Bauman Moscow State Technical University, Department of Applied Mathematics, 2nd Baumanskaya Str. 5/1, 105005, Moscow; Russian Federation, parshin@ipmnet.ru 
continues to change its stress-strain state even if the additive formation process stops. However, the study of additive processes with application of such materials is relevant from the point of view of engineering.

\section{The mechanical problem in question}

We consider a significantly high axisymmetric conical column being additively built up with use of the isotropic viscoelastic ageing material subordinated to the following constitutive equation:

$$
\mathbf{T}^{\circ}(\mathbf{r}, t)=2 \mathbf{E}(\mathbf{r}, t)+\frac{2 v}{1-2 v} \mathbf{1 1} \cdot \mathbf{E}(\mathbf{r}, t) .
$$

Here $\mathbf{T}$ is the stress tensor, $\mathbf{E}$ is the small strain tensor, $\mathbf{r}$ is the radius-vector of an arbitrary point of the considered solid, $t$ is the current time calculated from the material origination instant, $\mathbf{1}$ is the unit tensor of the second rank, $v=$ const is the Poisson's ratio for elastic and creep strain, and ( $)^{\circ}$ is the Volterra's integral operator of viscoelasticity defined as follows (Manzhirov, 1995):

$$
\mathbf{T}^{\circ}(\mathbf{r}, t)=\frac{\mathbf{T}(\mathbf{r}, t)}{G(t)}-\int_{\tau_{0}(\mathbf{r})}^{t} \frac{\mathbf{T}(\mathbf{r}, \tau)}{G(\tau)} K(t, \tau) d \tau,
$$

where $G(t)$ is the elastic shear modulus increasing with time due to material ageing,

$$
K(t, \tau)=G(\tau) \frac{\partial}{\partial \tau}\left[\frac{1}{G(\tau)}+\omega(t, \tau)\right]
$$

is the creep kernel, $t \geq \tau$ (Arutyunyan, 1966), with the material function $\omega(t, \tau)$ being the creep measure for simple shear and approximating respective experimental creep curves for different loading moments, and the time instant $\tau_{0}(\mathbf{r})$ is the instant of occurrence of stresses at the solid point $\mathbf{r}$. Remark that the here used material relations are widely employed to describe the mechanical behavior of various natural and artificial stones (in particular, concrete), polymers, soils, ice, wood. For example, experimental creep curves for some kinds of concrete can be borrowed from Ross (1958).

The considered column is bearing a time-variable external central axial force $F(t)$ applied to its upper end throughout the entire building process and assumed positive when compressing. The force begins to act at the time moment $t=t_{0}$ on a conical preform made stress-free in advance of the considered material.

At the moment $t=t_{1} \geq t_{0}$ the process of gradual building up the considered column by means of its axisymmetric augmenting is starting. Augmenting is implemented via layer-by-layer adding the additional material, being identical to the material of the preform and initially stress-free, to the column side surface. The built up column maintains a conical shape at each moment of the augmenting process.

Let the column bearing end be denoted by $\Omega(t)$. The time-dependence of this flat circular surface, i.e. increasing its radius in time, is caused by expansion of the column bearing end due to the additional material influx to the column side surface. We agree to put the origin of each point radius-vector $\mathbf{r}$ at the geometrical centre of the bearing end $\Omega(t)$ of the column. The time-varying side surface of the being augmented column will be denoted by $\Sigma(t)$. It is a conical surface with the arbitrarily changing slope angle, moving in space in the normal direction due to the additional material influx to the column.

The augmenting process is supposed to be piecewise continuous. That is it consists of arbitrary number $N$ of consecutive stages $t \in\left(t_{2 k-1}, t_{2 k}\right), k=\overline{1, N}$, at which an infinitely thin layer of additional material attaches to the column side surface each infinitely small period of time. These stages are interrupted with arbitrarily long pauses $t \in\left[t_{2 k}, t_{2 k+1}\right], k=\overline{1, N-1}$, when the influx of additional material to the column side surface is absent.

We investigate the stress-strain state evolution of the built up bearing column before, in course and after the stipulated piecewise continuous process of its layer-by-layer augmenting. We consider the case of small strain under quasi-static deformation assumption. 


\section{Mathematical formulation of the problem}

As the additional material is being loaded in the considered additive process just at the moment of its attaching to the column surface, we determine the function $\tau_{0}(\mathbf{r})$ in the following way:

$$
\tau_{0}(\mathbf{r})= \begin{cases}t_{0} & \text { inside the preform, } \\ \tau_{*}(\mathbf{r}) & \text { in the added material },\end{cases}
$$

where $\tau_{*}(\mathbf{r})$ is the distribution of the particles attaching moments.

The latter function can be used for calculation of the field of the external unit normal vectors $\mathbf{n}$ to the being augmented column side surface $\Sigma(t)$ :

$$
\mathbf{n}(\mathbf{r})=\frac{\nabla \tau_{*}(\mathbf{r})}{\left|\nabla \tau_{*}(\mathbf{r})\right|}, \quad \mathbf{r} \in \Sigma(t)
$$

Because of absence of any unstressed configuration for an additively built up solid, the kinematic description of its deformation process traditionally used in solid mechanics is not suitable for such a solid. But we can see that all particles of the newly added material move in space in the continuum composition after attaching to the surface of the being built up solid. So a sufficiently smooth velocities field $\mathbf{v}(\mathbf{r}, t)$ of the whole solid deforming movement is uniquely determined in the entire space region occupied by the considered instantly existing solid during its building up process. Therefore, we can give a mathematical formulation of the mechanical problem in question in velocities terms. For doing this we are to introduce the strain velocity tensor $\mathbf{D}(\mathbf{r}, t)$ and the operator stress velocity tensor $\mathbf{S}(\mathbf{r}, t)$ :

$$
\mathbf{D}=\frac{1}{2}\left(\nabla \mathbf{v}+\nabla \mathbf{v}^{\mathrm{T}}\right), \quad \mathbf{S}=\frac{\partial \mathbf{T}^{\circ}}{\partial t} .
$$

For these tensors the above given constitutive equation can be transformed to the form

$$
\mathbf{S}(\mathbf{r}, t)=2 \mathbf{D}(\mathbf{r}, t)+\frac{2 v}{1-2 v} \mathbf{1 1} \cdot \mathbf{D}(\mathbf{r}, t) .
$$

The considered being augmented solid obeys apparently the standard equilibrium equation

$$
\nabla \cdot \mathbf{T}(\mathbf{r}, t)=\mathbf{0} .
$$

It can be proved (Manzhirov, 1995) that the velocity analogue of this equation has the following simple form in the investigated case of being augmented solid loading:

$$
\nabla \cdot \mathbf{S}(\mathbf{r}, t)=\mathbf{0} \text {. }
$$

As we consider the unstressed additional material attached to the surface of the being built up column, we are to set the following initial condition in the added part of the column:

$$
\mathbf{T}\left(\mathbf{r}, \tau_{*}(\mathbf{r})\right)=\mathbf{0} .
$$

We can show that this non-classical initial condition together with the standard equilibrium equation lead in the considered mechanical problem to the following boundary condition for the operator stress velocity tensor on the column side surface moving in space due to continuous influx of additional material:

$$
\mathbf{n}(\mathbf{r}) \cdot \mathbf{S}(\mathbf{r}, t)=\mathbf{0}, \quad \mathbf{r} \in \Sigma(t), \quad t \in\left(t_{2 k-1}, t_{2 k}\right), \quad k=\overline{1, N} .
$$

This boundary condition holds good also in the pauses between the continuous augmening stages and after the last $(N$-th $)$ such stage, as the column side surface is free of stresses on the corresponding time segments.

It is undoubtedly to satisfy the loading conditions on the column bearing end $\Omega(t)$ in integral sense. In accordance with this thought, we have to set the following static boundary conditions on the bearing end:

$$
\int_{\Omega(t)} \mathbf{n}_{0} \cdot \mathbf{T}(\mathbf{r}, t) d S=-\mathbf{n}_{0} F(t), \quad \int_{\Omega(t)} \mathbf{r} \times\left[\mathbf{n}_{0} \cdot \mathbf{T}(\mathbf{r}, t)\right] d S=\mathbf{0} .
$$


Here $\mathbf{n}_{0}=$ const is the external unit normal vector to the bearing end $\Omega(t)$ of the column. This vector is directed opposite to the end force action direction if the force $F(t)$ is compressing.

\section{The way to solve the problem and conclusion}

The main intricacy to solve the stated problem is expanding in space the column bearing end $\Omega(t)$ due to influx of the additional material to its side surface $\Sigma(t)$. This hampers easy transformation of the above formulated integral static conditions to the operator stress velocity form. The staple attainment of the presented study lies in the proof of the following corollary of the specified integral conditions, taking place under all the assumptions made above:

$$
\int_{\Omega(t)} \mathbf{n}_{0} \cdot \mathbf{S}(\mathbf{r}, t) d S=-\mathbf{n}_{0} \frac{d}{d t}\left[\frac{F(t)}{G(t)}-\int_{t_{0}}^{t} \frac{F(\tau)}{G(\tau)} K(t, \tau) d \tau\right], \quad \int_{\Omega(t)} \mathbf{r} \times\left[\mathbf{n}_{0} \cdot \mathbf{S}(\mathbf{r}, t)\right] d S=\mathbf{0} .
$$

The essential limitation for deducing this corollary is the fact that the additional material is being attached stress-free to the side surface of the being built up column.

The written integral relations for the operator stress velocity tensor on the bearing end of the being augmented column allow to construct the closed-form analytical solution of the formulated mechanical problem. The constructed solution gives the opportunity to trace the stress-strain state evolution of a sufficiently high conical column bearing an axial loading on its upper end and being simultaneously built up by means of additive layer-by-layer surface augmenting with new viscoelastic ageing material over its side surface. The acting force magnitude, the radius of the increasing bearing end and the slope angle of the side surface are arbitrarily changing during the considered augmenting process.

\section{Acknowledgement}

This study is supported by the Ministry of Science and Higher Education of the Russian Federation (research theme state registration number AAAA-A17-117021310381-8) and, in part, by the Russian Foundation for Basic Research (grants Nos. 18-01-00920-a and 18-01-00770-a).

\section{References}

Manzhirov, A. V. and Parshin, D. A. (2015a) Arch structure erection by an additive manufacturing technology under the action of the gravity force. Mech. Solids, 50, 5, pp 559-570. DOI: 10.3103/S0025654415050076

Manzhirov, A. V. and Parshin, D. A. (2015b) Influence of the erection regime on the stress state of a viscoelastic arched structure erected by an additive technology under the force of gravity. Mech. Solids, 50, 6, pp 657-675. DOI: $10.3103 / \mathrm{S} 0025654415060072$

Manzhirov, A. V. and Parshin, D. A. (2016) Application of prestressed structural elements in the erection of heavy viscoelastic arched structures with the use of an additive technology. Mech. Solids, 51, 6, pp 692-700. DOI: $10.3103 / \mathrm{S} 0025654416060091$

Bychkov, P. S., Kozintsev, V. M., Manzhirov, A. V. and Popov, A. L. (2017) Determination of residual stresses in products in additive production by the layer-by-layer photopolymerization method. Mech. Solids, 52, 5, pp 524529. DOI: $10.3103 / \mathrm{S} 0025654417050077$

Parshin, D. A. (2017a) Analytic solution of the problem of additive formation of an inhomogeneous elastic spherical body in an arbitrary nonstationary central force field. Mech. Solids, 52, 5, pp 530-540. DOI: $10.3103 / \mathrm{S} 0025654417050089$

Parshin, D. A. (2017b) The technological stresses in a vaulted structure built up on a falsework. In: Mechanics for Materials and Technologies. Advanced Structured Materials, vol. 46 (Altenbach, H., Goldstein, R. and Murashkin, E. eds). Springer, Cham, pp 377-386. DOI: 10.1007/978-3-319-56050-2_22

Parshin, D. A. (2018) Impact of the rate of the additive process of forming a heavy structure deforming in creep on the development of its technological stresses. AIP Conf. Proc., 1959, 070029. DOI: 10.1063/1.5034704

Parshin, D. A. and Manzhirov, A. V. (2018) The mechanical problems on additive manufacturing of viscoelastic solids with integral conditions on a surface increasing in the growth process. J. Phys.: Conf. Ser., 991, 012063. DOI: $10.1088 / 1742-6596 / 991 / 1 / 012063$

Manzhirov, A. V. (1995) The general non-inertial initial-boundaryvalue problem for a viscoelastic ageing solid with piecewise-continuous accretion. Appl. Math. Mech., 59, 5, pp 805-816, DOI: 10.1016/0021-8928(95)00095-X

Arutyunyan, N. Kh. (1966) Some Problems in the Theory of Creep. Pergamon Press, Oxford.

Ross, A. D. (1958) Creep of concrete under variable stress. J. Amer. Concr. Inst., 29, 9, pp 739-758. 